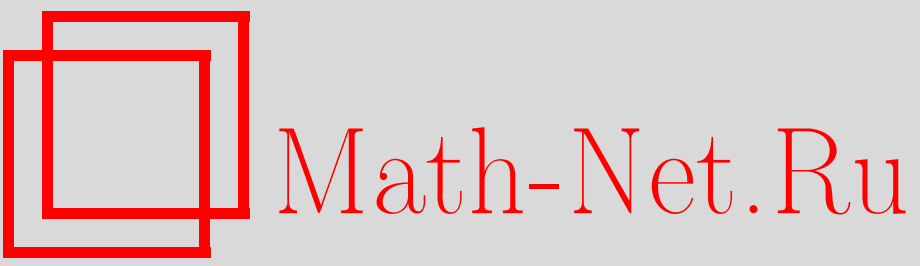

С. В. Козырев, Ультраметрическое пространство свободных когерентных состояний, ТМФ, 1997, том 110, номер 2, 334-336

DOI: https://doi.org/10.4213/tmf972

Использование Общероссийского математического портала Math-Net.Ru подразумевает, что вы прочитали и согласны с пользовательским соглашением

http://www.mathnet.ru/rus/agreement

Параметры загрузки:

IP: 54.237 .59 .107

26 апреля 2023 г., 11:53:24 
ТЕОРЕТИЧЕСКАЯ

И МАТЕМАТИЧЕСКАЯ

ФИЗИКА

Том 110, № 2

февраль, 1997

С.В. Козырев*

\section{УЛЬТРАМЕТРИЧЕСКОЕ ПРОСТРАНСТВО СВОБОДНЫХ КОГЕРЕНТНЫХ СОСТОЯНИЙ}

Определены свободные когерентные состояния для системы с двумя степенями свободы. Показано, что на множестве когерентных состояний, отвечающих одному собственному значению оператора уничтожения, естественным образом возникает 2-адический параметр.

\section{1. ВВЕДЕНИЕ}

В настоящей работе рассматриваются свободные когерентные состояния. Основным результатом работы является возникновение ультраметрического параметра на множестве когерентных состояний. Свободные перестановочные соотношения $A A^{\dagger}=1$ представляют интерес в связи с появлением таких соотношений для мастер-поля в квантовой хромодинамике (см. [1-3]). Свободные перестановочные соотношения и их обобщения обсуждаются в связи с некоммутативной теорией вероятности (см. $[4,5])$. Свободные перестановочные соотношения являются частным случаем $q$-деформированных соотношений

$$
A A^{\dagger}-q A^{\dagger} A=1
$$

для $q=0$ (см. обсуждение связи $q$-деформированных перестановочных соотношений с неархимедовой (ультраметрической) геометрией в работе [6]). Неархимедова математическая физика привлекает пристальное внимание и является предметом многих исследований (см. книгу [7]). Свободные когерентные состояния строятся в свободном фоковском пространстве. Свободным (или больцмановским) фоковским пространством $F$ над гильбертовым пространством $H$ называется тензорная алгебра

$$
F=\bigoplus_{n=0}^{\infty} H^{\otimes n}
$$

Операторы рождения и уничтожения определены как

$$
\begin{aligned}
A^{\dagger}(f) f_{1} \otimes \cdots \otimes f_{n} & =f \otimes f_{1} \otimes \cdots \otimes f_{n}, \\
A(f) f_{1} \otimes \cdots \otimes f_{n} & =\left\langle f, f_{1}\right\rangle f_{2} \otimes \cdots \otimes f_{n},
\end{aligned}
$$

* Институт химической физики РАН 
где $\langle f, g\rangle$ - скалярное произведение в гильбертовом пространстве $H$. Мы будем рассматривать случай $H=C \oplus C$. При этом имеются два оператора рождения $A_{0}^{\dagger}, A_{1}^{\dagger}$ и вакуумный вектор $\Omega$ :

$$
A_{i} \Omega=0,
$$

и соотношения

$$
A_{i} A_{j}^{*}=\delta_{i j}
$$

\section{2. СВОБОДНЫЕ КОГЕРЕНТНЫЕ СОСТОЯНИЯ}

Случай свободных когерентных состояний для нескольких степеней свободы отличается от случая бозонных когерентных состояний отсутствием перестановочности операторов рождения. Оказывается, что это приводит к новому эффекту. На множестве когерентных состояний возникает ультраметрический параметр. Свободные когерентные состояния строятся следуюшим образом.

Рассмотрим свободное фоковское пространство над двумерным евклидовым пространством. Это свободное фоковское пространство порождено свободными операторами рождения $A_{0}^{\dagger}, A_{1}^{\dagger}$ с вакуумного вектора $\Omega$. Введем положительные константы $\gamma_{0}, \gamma_{1}$, $0<\gamma_{i}<1$. Рассмотрим полубесконечную строку индексов $U=u_{1} u_{2} u_{3} \ldots$. Назовем свободным когерентным состоянием $X_{U}$ ряд $X_{U}=\sum_{k=0}^{\infty} X_{k}$, где $X_{0}=\Omega$-вакуум, $X_{k}=\gamma_{u_{k}} A_{u_{k}}^{\dagger} X_{k-1}$. Тогда имеем

$$
\left(\gamma_{0}^{-1} A_{0}+\gamma_{1}^{-1} A_{1}\right) X_{U}=X_{U} \quad \forall U
$$

Свободные когерентные состояния являются собственными векторами оператора уничтожения

$$
\gamma_{0}^{-1} A_{0}+\gamma_{1}^{-1} A_{1}
$$

отвечающими собственному значению 1 . Вырождение этого собственного пространства параметризуется полубесконечными строками из 0 и 1. На самом деле эти строки естественно считать 2-адическими числами. Чтобы увидеть это, введем на свободных когерентных состояниях метрику следуюшим образом.

Пусть $U, V$ суть произвольные строки. Тогда эти строки совпадают вплоть до элемента с номером $k$ включительно. Пусть $X_{U}, X_{V}$ - соответствующие свободные когерентные состояния. Введем следующим образом метрику $\rho$ :

$$
\rho\left(X_{U}, X_{V}\right)=\left\|X_{k}\right\|=\prod_{i=1}^{k} \gamma_{u_{i}}
$$

т.е. заселенность последнего совпадающего уровня $X_{U}$ и $X_{V}$. Метрика $\rho$ является ультраметрической и имеет явный физический смысл. В том случае, если $\gamma_{0}=\gamma_{1}=\gamma$, $\rho\left(X_{U}, X_{V}\right)=\gamma^{k}$ есть метрика на 2-адическом диске.

Сравним метрику $\rho$ с обычной метрикой $\tau$ скалярного произведения в свободном фоковском пространстве. Нетрудно видеть, что

$$
\tau\left(X_{U}, X_{V}\right)=\left\|X_{k}\right\|\left(\sum_{i=k+1}^{\infty}\left(\prod_{j=k+1}^{i} \gamma_{u_{j}}^{2}+\prod_{j=k+1}^{i} \gamma_{v_{j}}^{2}\right)\right)^{\frac{1}{2}}
$$


Будем считать, что $\gamma_{0}<\gamma_{1}$. Тогда получим

$$
\rho\left(\frac{2 \gamma_{0}^{2}}{1-\gamma_{0}^{2}}\right)^{\frac{1}{2}} \leq \tau \leq \rho\left(\frac{2 \gamma_{1}^{2}}{1-\gamma_{1}^{2}}\right)^{\frac{1}{2}},
$$

и метрики $\tau$ и $\rho$ порождают на пространстве свободных когерентных состояний эквивалентные топологии.

Благодарности. Автор благодарен И.В.Воловичу за обсуждения. Работа вьполнена при поддержке грантов J2R100 и JJ1100 Международного научного фонда и правительства Российской Федерации.

\section{Список литературы}

[1] I. Ya. Arefeva, I. V. Volovich. The Master Field for QCD and $q$-deformed Quantum Field Theory: Preprint SMI-25-95.

[2] R. Gopakumar, D. Gross. Nucl. Phys. 1995. V. B451. P. 379.

[3] M. R. Douglas, M. Li. Phys. Lett. 1995. V. B348. P. 360.

[4] L. Accardi, Y. G. Lu. Vigner semicircle low in quantum electrodynamics: Preprint of Volterra Center, № 126, 1992.

[5] H. Maassen. J.Funct.Anal. 1992. V. 106. P. 409.

[6] I. Ya. Arefeva, I. V. Volovich. Phys. Lett. B. 1991. V. 268. P. 179.

[7] В.С Владимиров, И. В. Волович, Е.И. Зеленов. р-Адический анализ и математическая физика. М.: Наука, 1994.

Поступила в редакцию 18.IV.1996 г.

\section{S. V. Kozyrev}

\section{ULTRAMETRIC SPACE OF FREE COHERENT STATES}

Free coherent states for a system of two degrees of freedom are defined. 2-adic parameter on the set of coherent states correspondent to eigenvalue of operator of annihilation is constructed. 